\title{
TITLE:
}

\section{Interrelations of Quantum- Mechanical Quantities Concerning Chemical Reactivity of Conjugated Molecules}

\section{$\operatorname{AUTHOR}(\mathrm{S}):$}

Fukui, Ken-ichi; Yonezawa, Teijiro; Nagata, Chikayoshi

\section{CITATION:}

Fukui, Ken-ichi ... [et al]. Interrelations of Quantum-Mechanical Quantities Concerning Chemical Reactivity of Conjugated Molecules. Bulletin of the Institute for Chemical Research, Kyoto University 1958, 35(5-6): 149-149

\section{ISSUE DATE:}

1958-03-30

URL:

http://hdl.handle.net/2433/75612

RIGHT: 


\title{
Interrelations of Quantum-Mechanical Quantities Concerning Chemical Reactivity of Conjugated Molecules
}

\author{
Ken-ichi Fukui, Teijiro Yonezawa and Chikayoshi NaGATA \\ (Kodama Laboratory)
}

J. Chem. Phys. 26, 831 (1957)

\begin{abstract}
It is attempted to disclose the interrelations underlying the parallelism found in several quantum-mechanical methods previously proposed to explain chemical reactivity in conjugated molecules. This is carried out mathematically in alternant hydrocarbons and hetero-alternant molecules mainly by the use of Coulson's integral formulas. In an alternant hydrocarbon, provided a condition is satisfied between minors of the secular determinant, the orders of reactivity of each position predicted by the self-polarizability, free valence, superdelocalizability, Wheland's localization energy, and Dewar's approximate localization energy by NBMO are shown to be in domplete agreement with one another. The relation of the frontier electron density and superdelocalizability is discussed. It is also demonstrated that a nonperturbation treatment for the so-called static method leads us to the same conclusion as the perturbation treatment. The parallelism of various quantities is discussed in their relation to the effect of heteroatom in heteroalternant molecules.
\end{abstract}

\section{Electron Microscopic Studies on Fillers for Rubber. (IV)}

\author{
State of Rubber-Filler System at the Hlongation \\ Eiji Surto and Masafumi A.rakawa \\ (Suito Laboratory) \\ Hiroshi Hasegawa and Yonemasa Furuzawa \\ (Research Laboratory, Shiraishi Kogyo Co., Ltd.
}

J. Soc. Rubber Ind., Japan, (Nippon Gomu Kyokaishi) 30, 251 (1957)

The rubber samples loaded with various fillers were elongated 200, 400, $600 \%$ by Martens-Schopper machine respectively, and after two hours standing the samples were prepared by the methylmethacryl-SiO two-step casting replica method of electron microscopy. The film was prepared from sticky liquor obtained by dissolving polymethacryl in acetone. The liquor was spread over the surface of the sample, and after drying, it was removed from the surface. Swelling or any other changes due to the acetone solution were not recognized on the sample. A.fter the $\mathrm{Cr}$-shadowing, SiO was evaporated on this film, and then the original film was dissolved in the solvent.

Two kinds of carbon black (fine thermal type), three kinds of calcium carbonate, basic magnesium carbonate and clay were used as filler. 